\title{
Primary care follow up of patients discharged from the emergency department: a retrospective study Shlomo Vinker*1,2, Eliezer Kitai ${ }^{2}$ Yaacov Or ${ }^{3}$ and Sasson Nakar ${ }^{1,2}$
}

Address: ${ }^{1}$ The Department of Family Medicine, Clalit Health Services, Central District, Rishon Lezion, Israel, ${ }^{2}$ The Family Medicine Department, Sackler Faculty of Medicine, Tel Aviv University, Tel Aviv, Israel and ${ }^{3}$ Emergency Department, Kaplan Medical Center Rehovot, Affiliated with the Hebrew University and Haddasa Faculty of Medicine, Rehovot, Israel

Email: Shlomo Vinker* - vinker01@inter.net.il; Eliezer Kitai - ekitaee@leumit.co.il; Yaacov Or - jacky_o@clalit.org.il;

Sasson Nakar - snakar@post.tau.ac.il

* Corresponding author

Published: 07 August 2004

BMC Family Practice 2004, 5:16 doi:10.1186/147|-2296-5-16
Received: 12 March 2004

Accepted: 07 August 2004

This article is available from: http://www.biomedcentral.com/147I-2296/5/16

(C) 2004 Shlomo et al; licensee BioMed Central Ltd.

This is an open-access article distributed under the terms of the Creative Commons Attribution License (http://creativecommons.org/licenses/by/2.0), which permits unrestricted use, distribution, and reproduction in any medium, provided the original work is properly cited.

\begin{abstract}
Background: The visit to the emergency department (ED) constitutes a brief, yet an important point in the continuum of medical care. The aim of our study was to evaluate the continuity of care of adult ED visitors.

Methods: We retrospectively reviewed all ED discharge summaries for over a month 's period. The ED chart, referral letter and the patient's primary care file were reviewed. Data collected included: age, gender, date and hour of ED visit, documentation of ED referral and ED discharge letter in the primary care file.

Results: 359 visits were eligible for the study. $192(53.5 \%)$ of the patients were women, average age $54.1 \pm 18.7$ years (mean \pm SD). $214(59.6 \%)$ of the visits were during working hours of primary care clinics ("working hours"), while the rest were "out of hours" visits. Only 196 (54.6\%) of patients had a referral letter, usually from their family physician. A third (7I/2/4) of "working hours" visits were self referrals, the rate rose to 63.5\% (92/145) of "out of hours" visits ( $P<$ $0.000 \mathrm{I})$. The ED discharge letter was found in $50 \%$ (179/359) of the primary care files. A follow-up visit was documented in only $31 \%$ ( $1 \mathrm{I} / 359)$. Neither follow up visit nor discharge letter were found in $43 \%$ of the files (I53/359).

Conclusions: We have found a high rate of ED self referrals throughout the day together with low documentation rates of ED visits in the primary care charts. Our findings point to a poor continuity of care of ED attendees.
\end{abstract}

\section{Background}

The emergency department (ED) is intended to treat medical urgencies or emergencies, but a large proportion of visits are due to problems that could be treated in the primary care setting $[1,2]$. ED services are available 24 hours a day while primary care facilities have limited service hours. In the Israeli health system patients can be referred to the ED by their family practitioner, or by other community health providers, or be self referred. Recently many out of hours community based services have been established but without a significant reduction in visiting rates to in-hospital ED. 
The visit to the ED constitutes a brief, yet an important point in the continuum of medical care. In today's era of cost effectiveness and increasingly competent primary care physicians, ambulatory investigation, treatment and follow-up have largely replaced prolonged and costly hospitalizations $[3,4]$. The ED visit however, remains a crossroad which may mark a sudden change in the patient's medical condition. In many cases it may result in introducing new medications, withdrawing others and recommendation of further investigations. The family practitioner is the one expected to coordinate and carry out the treatment and follow-up. The new information given from the ED should be effectively delivered to the family practitioner, the modality usually used is the discharge letter.

The continuation of treatment between hospital departments and the primary care physician had been issued in several studies using discharge letters audit [5-7]. Raval et. al. assessed the adequacy of the discharge summary in reporting important investigative results and future management plans in patients hospitalized and discharged with a diagnosis of heart failure [5]. They found substantial inadequacies in communicating to the community physician that may have implications for continuity of care and subsequent clinical outcome. Wilson et. al. examined the reliability, effectiveness, accuracy and timeliness of hospital to general practitioner information transfer by discharge summaries. In a retrospective audit of 569 patient discharge summaries and related medical records they found that summaries written for patients discharged from hospital were estimated to be received by the patient-nominated general practitioner in $27.1 \%$ of cases [6]. Bolton et al assessed the quality of communications between hospitals and general practitioners. The general practitioner's(GP) name was recorded in $88 \%$ of audited records. Few inaccuracies were detected in the medications recorded in the discharge summaries, and on contrary to Wilson et al $77 \%$ of discharge summaries were received by the GP [7]. The continuity of care between the ED and the primary care physician had been assessed for children with asthma [8] but we did not find data about the continuity of care for adults.

To evaluate the continuity of care after ED visits, we evaluated the ED referral and discharge letters, their content, and the documentation of the ED visit in the patients' primary care files. We have focused on discharges from the internal medicine ED. We expected that in these cases the patients would be followed up by their family practitioner.

\section{Methods}

The study was conducted in the district medical center (Kaplan), serving more than 500,000 inhabitants, and in
12 primary care clinics (32 family practitioners), of The Clalit Health Services in the Rehovot region, Israel.

In Israel the entire population have a national health insurance by law and each citizen can choose to be a member of one of four HMOs. Every member of the Clalit Health Services, the largest HMO in Israel, is registered to a single family physician, and have a medical record in his physician's clinic. Visits to the emergency department are regulated in the national health insurance law. A referral by a physician or by ambulance is free of charge, but this referral should be with a referral letter and not by a phone call to the ED or to the patient. A self referral may cost the patient a co-payment of up to 100 USD.

We reviewed retrospectively all the charts of the ED visits for a period of one month, excluding the visits to the pediatric and the gynecologic-obstetrics EDs (see flow-chart 1). 5,898 visits documented that month, resulted in 4,256 discharges and 1,642 hospitalizations. There were 1,564 discharges from the general ED. Trauma, surgery and orthopedics accounted 2,209 discharges and the rest 483 were from other specialties (ophthalmology, ENT, dermatology etc.).

Inclusion criteria were: visit to the general ED, age above 18 years, discharge to the community (not hospitalized) at that visit, living and getting medical care in a family medicine group practice in the Rehovot region. Visits due to accidents, trauma, surgery, orthopedics, ENT, ophthalmology and other specialities were excluded from the study. The 1,564 discharges from the general ED were reviewed and 359 were found to be eligible to this study.

Two physicians reviewed independently each ED medical chart. Data extracted included: age and gender of the patient, attendance date and hour, self referral, or a referral by a physician and the final diagnosis in the discharge letter. In cases of referrals the content and format of the referral letter were assessed, including: hand writing quality and whether the referring physician referred the patient with a specific question (for example: rule out new onset angina pectoris, suspected pneumonia, please make a chest $\mathrm{X}$ ray etc.).

Continuity of communication and care: The primary care files of ED visitors were retrieved and checked for the existence of the ED discharge letter and comments about the visit in the follow-up chart. If the discharge letter and / or any comment on the ED visit in the follow-up chart had been found the case was defined as "a case with good continuity of care". 
The cases in which the family physician was the referring physician we looked for documentation of the encounter prior to ED attendance.

Visits to the ED were divided into "working hours" visits - when the visit took place during working hours of primary care clinics in the community (Sunday to Thursday from 08:00 to 20:00, and Friday 08:00 to 14:00), and "out of hours" visits when primary care clinics in the community are closed (from 20:00 to 08:00 weekdays, and weekends from 14:00 on Friday to 08:00 on the following Sunday).

A referral letter was defined as "any document written by a medical authority in the community prior to the index ED visit", including referrals from family practitioners, other practitioners in the community and arrival by an ambulance.

A recurrent visit to the ED was defined as a patient's visit to ED within less than two weeks from a previous visit with the same complaint, when in both cases the patient was not hospitalized.

Diagnoses at discharge were coded for a specific diagnosis and for the system involved according to the ICPC coding system.

Statistical analysis: Data was analyzed using distribution analysis and $\chi^{2}$ tests to investigate the association between categorized variables. Student's t tests were used to analyze continuous variables. The analysis was performed using the SPSS package.

\section{Results}

During the study period there were 359 ED visits that were eligible to be included in the study (table 1, flow chart 1 ). $.214(59.6 \%)$ visited the ED during the "working hours" of primary care clinics, 28 (7.8\% of all visits) were recurrent visits to the ED.

Out of all ED visits only 196 (54.6\%) patients had a referral letter, the rest were self-referrals. Referral letters were mainly from the family practitioner (147/196, 75\%), 14 (7\%) from other practitioners in the community, and 35 (18\%) of referrals were by ambulance. The referral letters from the community were legible in $43.4 \%(70 / 161)$, $46.5 \%$ (75/161) were barely legible and 10\% (16/161) illegible. In only $25 / 161$ letters (15.7\% of the referrals) a specific question was asked by the referring physician and in another $32(20.2 \%)$ there was only a general question.

The main diagnostic groups according to the ICPC were: respiratory $(15.7 \%)$, digestive system $(18.1 \%)$, musculoskeletal (15.2\%) and cardio-vascular (11\%). In $9.6 \%$ of the cases the discharge letter did not contain a specific diagnosis and the diagnosis fell in the "general" category. The most common specific diagnoses were: chest pain $(5.9 \%)$, abdominal pain $(3.9 \%)$, other respiratory tract infections $(3.7 \%)$, asthma (3.1\%), back pain $(2.8 \%)$, COPD exacerbation $(2.8 \%)$ headache $(2.5 \%)$, nephrolithiasis $(2.5 \%)$, vertigo or dizziness $(2.5 \%)$ and gastroenteritis $(2.3 \%)$.

The "out of hours" visitors tended to be younger (52.2 \pm 17.5 vs. $55.4 \pm 19.5, \mathrm{p}=\mathrm{NS}$ ) (table 1 ). A third of the "working hours" visits $(71 / 214)$ were self referrals as opposed to $63.5 \%$ (92/145) of "out of hours" visits ( $\mathrm{p}<$ $0.0001)$. Table 2 compares the referring practitioners according to ED visiting hours.

In 147 cases the reffering physician was the family physician, documentation of the ED referral was found in $32 \%$ (47/147) of primary care files. The ED discharge letter was found in $50 \%(179 / 359)$ of the primary care files. A follow-up visit was documented in only $31 \%(111 / 359)$. Neither follow up visits nor discharge letters were found in $43 \%$ of the files (153/359). No associations between clinic characteristics (size, place) or family practitioner qualification and ED visit documentation was found.

\section{Discussion}

The Emergency Department (ED) acts as a link between community and hospital based medicine. In Israel a patient who needs non elective admission to a hospital unit must pass through the $\mathrm{ED}$, either with a referral note from a medical practitioner, or as a self referral. Most ED visits, however, do not result in hospitalization, and many could be regarded as primary health care problems $[1,2,9]$. These patients are discharged directly from the ED to the community and further care of the family practitioner. A visit to the ED is generally not prompted by a benign complaint; The most common reasons for referral include, chest pain, asthma exacerbations and nephrolithiasis, subsequent follow up by the family practitioner can be vital. It was found that most children do not have outpatient follow-up after an ED asthma visit [8]. However, those patients that present for outpatient follow-up have an increased likelihood for repeat ED asthma visits, and this visit should be a key opportunity to prevent future ED asthma visits.

The increasing role played by the ED in treating primary care problems has been discussed in a number of recent articles [9-11].

One aspect, which is important to the ED team, is the logistics and manpower needed to optimize the treatment of these non-urgent patients in ways that will not interfere with emergencies yet providing them adequate care. It is 
Table I: Data on 359 visitis to the Emergency Department

\begin{tabular}{|c|c|c|c|c|}
\hline & All visits (359) & Referral letter (196) & Self referral (163) & P Value* \\
\hline \multicolumn{5}{|l|}{ Gender } \\
\hline Women - & $192(53.5 \%)$ & $106(54 \%)$ & $86(52.7 \%)$ & NS \\
\hline Men - & $167(46.5 \%)$ & $96(46 \%)$ & 77 (47.3\%) & \\
\hline Age (years, mean $\pm S D)$ & $54.1 \pm 18.7$ & $55.1 \pm 19.0$ & $52.9 \pm 18.4$ & NS \\
\hline \multicolumn{5}{|l|}{ Age distribution } \\
\hline$<45$ & 127 (35.4\%) & $68(34.7 \%)$ & $59(36.2 \%)$ & NS \\
\hline $46-65$ & $105(29.3 \%)$ & $53(27 \%)$ & $52(31.9 \%)$ & \\
\hline $66-75$ & $63(17.5 \%)$ & 38 (19.4\%) & $25(15.3 \%)$ & \\
\hline$>75$ & $64(17.8 \%)$ & $37(18.9 \%)$ & $27(16.6 \%)$ & \\
\hline \multicolumn{5}{|l|}{ Visit time } \\
\hline Working hours - & $214(59.6 \%)$ & 143 (73\%) & 71 (43.6\%) & $<0.0001$ \\
\hline Out of hours - & 145 (40.4\%) & $53(27 \%)$ & $92(56.4 \%)$ & \\
\hline
\end{tabular}

* _ p Value for comparison between patients with referral letter and self referrals

Table 2: Comparison between the source of referral, in 196 visits according to ED visit hours*

\begin{tabular}{lccc}
\hline The referring practitioner & "Working hours"** & "Out of hours" & Total \\
\hline The Family practitioner & $125(87.5 \%)$ & $22(41.5 \%)$ & 147 \\
By ambulance & $16(11 \%)$ & $19(36 \%)$ & 35 \\
Other practitioner*** & $2(1.5 \%)$ & $12(22.5 \%)$ & 14 \\
Total & $143(100 \%)$ & $53(100 \%)$ & 196 \\
\hline
\end{tabular}

$*_{-} \mathrm{p}<0.0001 * *-$ "working hours" visits - when the visit was during the working hours of primary care clinics in the community $* * *-$ private practitioners, and out of hours commmunity emergency clinics

unclear whether the capability and quality of primary care services in the ED should be improved and compete with the community family physicians. This is true especially in Israel where there is a universal national health insurance and every patient can have a personal family practitioner.

The continuity of comprehensive management is expected from the family practitioner, and is gaining importance nowadays [12]. To achieve this goal the communication between health care providers who treat the patient is mandatory. In the case of the ED visit, where we found many self referrals and referrals from other physicians, it becomes even more important.

The modes of communication are the referral letter and the discharge letter. We have found that the referral letter can be improved both in style (printed instead of illegible hand writing) and content (the referring physician should define and clarify the reasons for referral and his expectations). These problems exist in discharge letters as well [13].
Documentation in the primary care file was poor, only one third of referrals were documented and less than $60 \%$ of discharges. This figure is between the $27 \%-77 \%$ that was found by others $[6,7]$. A possible bias is that some follow-up visits were to specialists. But in the case of discharge from the general medicine ED we presume that most patients were advised to return to their family physicians.

It is well known that medical notes are poor in other areas, Miller et al [14] found documentation of only $15 \%$ of prescriptions given by family practitioners. They explained one of the causes for this discrepancy as the need of double writing (both the prescription itself and in the medical notes). By introducing carbon copy prescriptions, they achieved an $82 \%$ documentation rates in patients' files. Opila [15] found documentation in out patient medical records greatly improved after employing quality control and a feedback system.

With the introduction of computerized medical files in primary care clinics in our region, the need of "double writing" will disappear, and this in turn should dramati- 


\section{All visits to the ED (1 month period) $\quad 5,898$}

Discharged to the community

Discharges from trauma, surgery,

orthopedics ED

2,209

Discharges from other* ED $\quad \underline{-483}$

specialities

Discharges from general ED $\quad 1,564$

Living and getting medical care in a $\quad 359$ family medicine group practices in the Rehovot region

* Other specialities: ENT, ophthalmology, dermatology etc. 
cally improve documentation rate of referrals and discharges to the ED; particularly if a computerized reminder system is used to encourage follow up of referrals by the family practitioner.

\section{Limitations}

Israeli health care system works in regard to ED use quite different from the US and other countries. Likewhise these results may not automatically be generalized to other health care systems. This study described the written communication between the emergency department and the primary care physician, which is the first and mandatory step in establishing continuation of care. This is only one of the four dimensions of continuity of care in family practice: chronological, geographical, interdisciplinary, and interpersonal [16]. Each of these dimensions may influence the quality of care and be evaluated and studied. Further study is needed to prove the link between documantion of ED visit and good contuniuity of care. Large scale prospective intervention studies are needed to prove that continuity of care between ED and the primary care physician improves outcome and saves money.

\section{Conclusion}

ED visits may have important implications for the patient and his family practitioner. The high rate of ED self referrals together with low documentation rates of ED visits in the primary care charts result in poor continuity of care of ED visitors.

\section{Competing interests}

None declared.

\section{Authors' contributions}

All authors read and approved the final manuscript.

VS Conceived and designed the study, participated in the collection, analysis and interpretation of data and drafted the manuscript. KE Participated in the statistical analysis, interpretation of data and draft of the manuscript.OY participated in the design of the study, data collection and interpretetio hand draft of the manuscript. SN participated in the design of the study, interpretation of data and draft of the manuscript. All authors have read and approved the final manuscript.

\section{Acknowledgments}

We want to thank Dr Gavriel Plotkin for his participation in the data collection.

\section{References}

I. Dale J, Green J, Reid F, Glucksman E: Primary care in the accident and emergency department: I prospective identification of patients. $B M J$ I 995, 3 I I:423-426.

2. Dale J, Lang H, Roberts JA, Green J, Glucksman E: Primary care in the accident and emergency department: II comparison of general practitioners and hospital doctors. BM] 1995 , 3 I I:427-430.

3. Steiner JF, Feinberg LE, Kramer AM, Byyny RL: Changing patterns of disease on an inpatient medical service: $1961-1962$ to 198I-I 982. Am J Med 1987, 83:33I-335.

4. Boehm R, Suissa M, Glick SM: Changes in the nature of inpatient medical services - impact on medical education and patient care. Isr J Med Sci 1994, 30:125-I29.

5. Raval AN, Marchiori GE, Arnold JM: Improving the continuity of care following discharge of patients hospitalized with heart failure: is the discharge summary adequate? Can J Cardiol 2003, 19:365-70.

6. Wilson S, Ruscoe W, Chapman M, Miller R: General practitionerhospital communications: a review of discharge summaries. I Qual Clin Pract 200I, 21:104-8.

7. Bolton P, Mira M, Kennedy P, Lahra MM: The quality of communication between hospitals and general practitioners: an assessment. J Qual Clin Pract 1998, 18:24I-7.

8. Cabana MD, Bruckman D, Bratton SL, Kemper AR, Clark NM: Association between outpatient follow-up and pediatric emergency department asthma visits. J Asthma 2003, 40:74I-9.

9. Young GP, Wagner MB, Kellermann AL, Ellis J, Bouley D, for the 24 Hours in the ED Study Group: Ambulatory visits to hospital emergency departments, patterns and reasons for use. JAMA 1996, 276:460-465.

10. Adams SL, Fontanarosa PB: Triage of ambulatory patients. JAMA 1996, 276:493-494.

II. Dale J, Green J, Reid F, Glucksman E, Higgs R: Cost effectiveness of treating primary care patients in accident and emergency: a comparison between general practitioners, senior house officers, and registrars. $B M]$ | 995, 3 | 2: |340-|344.

12. Bowman MA, Nicklin DA: New developments in family medicine. JAMA 1998, 279: |437-1438.

13. Zalewski S, Lederer J: Evaluation of emergency room reply letters. Harefuah 1984, 107:342-344.

14. Miller LG, Matson CC, Rogers JC: Improving prescription documentation in the ambulatory setting. Fam Pract Res J 1992, | 2:42 I -429.

15. Opila DA: The impact of feedback to medical housestaff on chart documentation and quality of care in the outpatient setting. J Gen Intern Med 1 997, I 2:352-356.

16. Hennen BK: Continuity of care in family practice. Part I: dimensions of continuity. J Fam Pract 1975, 2:37I-2.

\section{Pre-publication history}

The pre-publication history for this paper can be accessed here:

http://www.biomedcentral.com/1471-2296/5/16/prepub

Publish with Bio Med Central and every scientist can read your work free of charge

"BioMed Central will be the most significant development for disseminating the results of biomedical research in our lifetime. "

Sir Paul Nurse, Cancer Research UK

Your research papers will be:

- available free of charge to the entire biomedical community

- peer reviewed and published immediately upon acceptance

- cited in PubMed and archived on PubMed Central

- yours - you keep the copyright

Submit your manuscript here:

http://www.biomedcentral.com/info/publishing_adv.asp
BioMedcentral 